\title{
Equivalência de Estímulos e Autismo: Uma Revisão de Estudos Empíricos ${ }^{1}$
}

\author{
Camila Graciella Santos Gomes \\ André Augusto Borges Varella \\ Deisy das Graças de Souza ${ }^{2}$ \\ Universidade Federal de São Carlos \\ Instituto Nacional de Ciência e Tecnologia sobre Comportamento, Cognição e Ensino
}

\begin{abstract}
RESUMO - O modelo de equivalência de estímulos oferece uma especificação operacional do comportamento simbólico e tem orientado o ensino e a verificação objetiva de repertórios novos. Pessoas com autismo, caracterizadas por apresentarem, entre outras alterações, graves déficits de linguagem, poderiam se beneficiar de estratégias baseadas neste modelo. Este artigo apresenta uma revisão de publicações de estudos empíricos com autistas, fundamentados no paradigma de equivalência. Os resultados mostraram sucesso de alguns participantes e fracasso de outros. Os fracassos parecem residir mais em dificuldades em aprender relações arbitrárias e menos na emergência de relações novas após aquisição da linha de base. O desafio para a área é desenvolver procedimentos que favoreçam a aprendizagem de relações arbitrárias e, consequentemente, a formação de classes de estímulos equivalentes.
\end{abstract}

Palavras-chave: comportamento simbólico; equivalência de estímulos; aprendizagem relacional; relações arbitrárias; autismo.

\section{Stimulus Equivalence and Autism: A Review of Empirical Studies}

\begin{abstract}
The model of stimulus equivalence offers an operational specification of symbolic behavior, thus orienting teaching strategies and allowing for objective measures of new behavioral repertoires. Autistic persons, often characterized by presenting, among other disabilities, severe language deficits, could benefit from strategies based on this model. This paper reviews the few empirical studies conducted with autistic participants, based on the stimulus equivalence paradigm. Some participants were successful, while others failed. The failures seem to be more related to difficulties in learning arbitrary relations rather than to the emergence of novel relations itself, after the baseline has been established. The challenge for the field is to develop procedures that are truly effective in establishing arbitrary relations and, consequently, the formation of equivalence classes.
\end{abstract}

Keywords: symbolic behavior; stimulus equivalence; relational learning; arbitrary relations; autism.

Compreender o meio social e interagir com outras pessoas envolve diversos comportamentos, entre eles habilidades que permitem entender códigos compartilhados socialmente que se encontram presentes na fala, na leitura, na escrita, em regras, em normas e em valores. Essas habilidades, que envolvem basicamente "compreender" ou "entender" códigos ou símbolos sociais, podem ser desenvolvidas a partir do estabelecimento de relações arbitrárias ou convencionais entre os símbolos e seus referentes, como no caso dos nomes atribuídos a objetos, em que o nome é o símbolo e o objeto concreto é o referente. Esse tipo de relação é arbitrária porque o símbolo (nome) não apresenta qualquer semelhança física com o referente (objeto) e varia de acordo com as convenções de cada comunidade verbal (de Rose, 1998; Dube, 1996).

A aprendizagem de relações entre símbolos e referentes, assim como a utilização desse tipo de relação em situações cotidianas, além de favorecer a interação social, oferece

1 Os autores agradecem a dois revisores anônimos pela leitura cuidadosa e pelas sugestões que tornaram melhor a versão final do artigo. Apoio: MCT/CNPq/FAPESP (Processos 573972/2008-7 e 08/57705-8).

2 Endereço para correspondência: Universidade Federal de São Carlos, Centro de Educação e Ciências Humanas. Laboratório de Estudos do Comportamento Humano (LECH), Rodovia Washington Luís, km 235, CEP: 13565-905, São Carlos, São Paulo - Brasil, Telefone: (16) 33518492.E-mails: camilagsg@uol.com.br, andreabvarella@gmail.com, ddgs@ufscar.br algumas vantagens em relação à utilização de objetos ou outros aspectos do mundo concreto, pois permite, em diversas situações, autonomia do símbolo em relação ao referente. Por exemplo, quando queremos contar algo a alguém, não é necessário mostrar objetos ou realizar as ações relatadas para que sejamos compreendidos; basta falar ou mesmo escrever. De maneira mais ampla, a capacidade de compartilhar ativamente de sistemas simbólicos complexos, que envolvem estímulos de naturezas diferentes e que afetam diferentes modalidades sensoriais, como aqueles desenvolvidos por humanos, está relacionada à linguagem ou comportamento verbal, definida nos termos da análise do comportamento (Catania, 1999).

Considerando-se a complexidade e a sofisticação dos sistemas simbólicos, pode-se imaginar que a construção destes sistemas não se deve apenas à aprendizagem direta de relações arbitrárias entre estímulos; é possível que, a partir da aprendizagem de algumas relações estabelecidas por ensino explícito, outras relações sejam derivadas das primeiras, sem terem sido diretamente ensinadas, ampliando as relações entre diversos estímulos diferentes. Desta forma, a aprendizagem de algumas relações condicionais arbitrárias entre estímulos pode constituir-se como base para o estabelecimento de outras relações entre símbolos e seus referentes que, por sua vez, é aspecto fundamental e necessário para a linguagem (Sidman, 1994). 
A concepção de linguagem como comportamento simbólico tem sido descrita por meio do paradigma da equivalência de estímulos, definido por Sidman (1971) e redefinido experimentalmente por Sidman e Tailby (1982). Para estes pesquisadores o comportamento simbólico pode ser compreendido por redes de relações arbitrárias entre estímulos; em uma rede, algumas relações são aprendidas diretamente, enquanto outras ocorrem por derivação, sem ensino direto, a partir da aprendizagem inicial.

De acordo com o modelo de Sidman, é possível simular as aprendizagens cotidianas ensinando uma criança a relacionar, por exemplo, um conjunto de estímulos como palavras ditadas (conjunto A), a outro conjunto de estímulos como figuras (conjunto B). Tal relação poderia ser ensinada da seguinte maneira: o professor ou experimentador dita a palavra "bolo" e a criança é ensinada a selecionar a figura de um bolo, entre outras figuras apresentadas simultaneamente. Em nova oportunidade, a palavra "suco" é ditada, mas apesar da figura do bolo estar disponível, a figura a ser selecionada deve ser correspondente à nova palavra ditada, neste caso, a figura do suco. Esta é uma tarefa de emparelhamento com o modelo típico (matching típico): a palavra ditada é o estímulo modelo e as figuras são estímulos de comparação ou de escolha. Para cada modelo, há apenas um estímulo de comparação correto e todos os outros estímulos de comparação são incorretos. Diferentes modelos se alternam ao longo de tentativas sucessivas. Em situação de ensino, as respostas de escolha são seguidas por consequências diferenciais, ou seja, respostas corretas são reforçadas e escolhas incorretas não são seguidas de reforço (de Rose, 2004; Dube, 1996; Saunders \& Green, 1999). Assim, são estabelecidas relações entre elementos do conjunto $\mathrm{A}$ e do conjunto $\mathrm{B}$ (relações $\mathrm{AB}$ ), no exemplo que vem sendo descrito. Da mesma forma, pode-se ensinar também relações entre outros conjuntos de estímulos, como por exemplo, entre o mesmo conjunto A (palavras ditadas) e um conjunto $C$, formado por palavras impressas (relação $\mathrm{AC})$. Após serem diretamente ensinadas, as relações AB e AC podem dar origem a outras relações não ensinadas. Ao mostrar para a criança uma figura do conjunto B (a figura de bolo, por exemplo) ela poderá selecionar, em meio a outras palavras impressas do conjunto $\mathrm{C}$, a palavra impressa bolo (relação BC) sem que tenha sido diretamente ensinada a fazê-lo. Da mesma forma, ao mostrar para a criança a palavra impressa bolo, ela poderá selecionar a figura de um bolo (relação CB). Esse tipo de relação que surge de forma indireta, a partir do ensino de outras relações, é denominada emergente. Considera-se que os estímulos dos conjuntos A, B e C (uma palavra falada, uma figura e uma palavra impressa, no exemplo) formam uma classe de equivalência se as relações entre eles apresentarem, simultaneamente, as propriedades de simetria, reflexividade e transitividade, por analogia com a Teoria dos Conjuntos da matemática (Sidman \& Tailby, 1982).

O estudo de Sidman e Cresson (1973) demonstra empiricamente a formação de classes de equivalência. Nesse trabalho, ensinou-se a jovens com retardo mental a escolher figuras (B) quando os nomes dessas figuras (A) eram ditados (AB) e a escolher palavras impressas (C) quando os nomes dessas palavras impressas (A) eram ditados (AC). Uma das propriedades das relações de equivalência pode ser inferida quando o participante é capaz de, frente a um estímulo modelo, selecionar um estímulo de comparação idêntico sem ter sido ensinado, demonstrando reflexividade. A simetria pode ser verificada quando se ensina uma relação, como por exemplo $\mathrm{AB}$, e a relação inversa $\mathrm{BA}$ emerge sem ter sido ensinada. A transitividade pode ser verificada quando, após treino de relações que possuem um estímulo em comum como no caso das relações AB e AC, que apresentam o estímulo A em comum, verifica-se a emergência da relação condicional $\mathrm{BC}$ sem que essa relação tenha sido diretamente ensinada. No caso do estudo descrito, a reflexividade foi verificada quando os participantes, após a aprendizagem da relação de identidade entre alguns estímulos, foram capazes de emparelhar outros estímulos idênticos sem terem sido diretamente ensinados; a simetria e a transitividade puderam ser verificadas pelos testes de relações emergentes entre figuras e palavras impressas, assim como o inverso (BC e CB).

$\mathrm{O}$ conceito de equivalência tem norteado a verificação objetiva e sistemática de repertórios novos, complexos, sob controle de relações simbólicas com diferentes populações: crianças com desenvolvimento típico (e.g., Pilgrim, Jackson, \& Galizio, 2000), deficientes mentais (e.g., Sidman, 1971; Sidman \& Cresson, 1973) e adultos (e.g., Green, Sigurdardottir, \& Saunders, 1991). Além disso, tornou-se um instrumento eficaz para o planejamento de ensino de habilidades de linguagem como a leitura e a escrita (e.g., de Rose, de Souza, \& Hanna, 1996;) e conceitos matemáticos (e.g., Prado \& de Rose, 1999).

A vantagem do ensino de habilidades que envolvem comportamento simbólico com base no paradigma da equivalência está no fato de que a metodologia utilizada favorece o importante processo de emergência de comportamentos novos a partir do ensino de algumas poucas relações, representando um aumento na produtividade do participante e uma economia no processo de ensino, uma vez que não é necessário ensinar todas as relações relevantes. Assim, o indivíduo passa a emitir comportamentos que não emitia, sem que estes tenham sido diretamente ensinados e que são mantidos mesmo sem serem diretamente reforçados (de Rose, 1998).

\section{Equivalência de estímulos e autismo}

Dentre todas as populações que poderiam se beneficiar de estratégias com base na formação de classes de estímulos equivalentes para o estabelecimento de comportamento simbólico encontram-se, em especial, pessoas com autismo, caracterizadas por apresentarem, entre outras alterações, déficits de interação social, de comunicação e linguagem e pela presença de padrões estereotipados de comportamentos. Autistas demonstram, de modo geral, dificuldades em interpretar o que observam, em dar sentido além do literal, em associar palavras ao seu significado, em compreender a linguagem falada, figuras de linguagem, ironias e conceitos abstratos, em utilizar a fala com função comunicativa e em generalizar a aprendizagem. Todas essas dificuldades, acrescidas da baixa emissão de brincadeira simbólica ou de faz-de-conta, estão diretamente relacionadas a alterações na linguagem e sugerem as limitações que esses aspectos podem 
trazer para a vida das pessoas com autismo e das que com elas convivem (APA, 2002).

Estudos publicados em periódicos científicos nacionais e internacionais da área, conduzidos com participantes autistas e fundamentados no paradigma da equivalência, são escassos. Até 2009, 38 anos após o trabalho clássico de Sidman (1971) e 56 anos após a definição inicial de autismo (Kanner, 1943), foram identificados nove artigos, seis dos quais na última década: Eikeseth e Smith (1992); Maguire, Stromer, Mackay e Demis (1994); Dube e McIlvane (1995); Carr, Wilkinson, Blackman e McIlvane (2000); LeBlanc, Miguel, Cumming, Goldsmith e Carr (2003); Vause, Martin, Yu, Marion e Sakko (2005); Barros, Lionello-DeNolf, Dube e McIlvane (2006); O'Connor, Rafferty, Barnes-Holmes e Barnes-Holmes (2009); Wilkinson, Rosenquist e McIlvane (2009).
É importante ressaltar que o autismo não é considerado um transtorno raro e as estimativas, embora contraditórias, apontam de um caso em cada 600-700 pessoas a um caso em cada 250 pessoas (Filipek e cols., 1999). Além disso, essa população tem sido beneficiada com estratégias de ensino intensivo, baseadas em tecnologias desenvolvidas pela análise do comportamento e reconhecidas por sua eficácia (Lovaas, 2003), inclusive para ensinar habilidades relacionadas à linguagem (Sundberg \& Partington, 1998)

A Tabela 1 apresenta os aspectos gerais de estudos publicados que se propuseram a documentar o desempenho de participantes com autismo na aprendizagem de relações arbitrárias entre estímulos e em testes de formação de classes de estímulos equivalentes.

Tabela 1. Aspectos Gerais dos Estudos sobre Equivalência de Estímulos com Participantes Autistas Publicados em Periódicos

\begin{tabular}{|c|c|c|c|c|c|c|}
\hline Estudos & $\begin{array}{c}\text { Número de } \\
\text { participantes }\end{array}$ & Estímulos & $\begin{array}{c}\text { Material / } \\
\text { equipamento } \\
\text { utilizado na } \\
\text { organização } \\
\text { das tarefas }\end{array}$ & $\begin{array}{l}\text { Procedimento de ensino de } \\
\text { relações arbitrárias }\end{array}$ & $\begin{array}{l}\text { Apren } \\
\text { Dificuldades } \\
\text { no ensino } \\
\text { de relações } \\
\text { arbitrárias }\end{array}$ & $\begin{array}{l}\text { Formação de } \\
\text { classes }\end{array}$ \\
\hline $\begin{array}{c}\text { Eikeseth \& } \\
\text { Smith (1992) }\end{array}$ & 4 & $\begin{array}{l}\text { Letras gregas maiús- } \\
\text { culas, minúsculas e os } \\
\text { nomes das letras gregas } \\
\text { impressos }\end{array}$ & Papel & $\begin{array}{l}\text { Matching simultâneo: um } \\
\text { modelo e dois comparações }\end{array}$ & Sim & $\begin{array}{l}\text { Sim, após } \\
\text { nomeação }\end{array}$ \\
\hline $\begin{array}{l}\text { Maguire e cols. } \\
\text { (1994) }\end{array}$ & 2 & $\begin{array}{c}\text { Figuras em preto e } \\
\text { branco e palavras } \\
\text { ditadas }\end{array}$ & $\begin{array}{l}\text { Papel e compu- } \\
\text { tador }\end{array}$ & $\begin{array}{l}\text { Matching simultâneo: um } \\
\text { modelo e dois comparações }\end{array}$ & Não & Não \\
\hline $\begin{array}{c}\text { Dube \& McIl- } \\
\text { vane (1995) }\end{array}$ & 2 & $\begin{array}{l}\text { Figuras abstratas e re- } \\
\text { forçadores comestíveis }\end{array}$ & Computador & $\begin{array}{l}\text { Discriminações simples e } m a \text { - } \\
\text { tching simultâneo: um modelo } \\
\text { e dois comparações (uso de } \\
\text { Fading e dica atrasada) }\end{array}$ & Sim & Não \\
\hline $\begin{array}{l}\text { Carr e cols. } \\
\qquad(2000)\end{array}$ & 2 & Figuras abstratas & Papel & $\begin{array}{l}\text { Matching simultâneo: um } \\
\text { modelo e dois comparações }\end{array}$ & Não & $\begin{array}{l}\text { Um partici- } \\
\text { pante }\end{array}$ \\
\hline $\begin{array}{l}\text { Leblanc e cols. } \\
\qquad(2003)\end{array}$ & 2 & $\begin{array}{l}\text { Mapas e nomes impres- } \\
\text { sos de estados e capitais }\end{array}$ & Papel & $\begin{array}{l}\text { Matching simultâneo: um } \\
\text { modelo e três comparações }\end{array}$ & Não & Sim \\
\hline $\begin{array}{l}\text { Vause e cols. } \\
\qquad(2005)\end{array}$ & 1 & $\begin{array}{l}\text { Figuras, palavras im- } \\
\text { pressas e símbolos }\end{array}$ & Papel & $\begin{array}{l}\text { Matching simultâneo: um mo- } \\
\text { delo, três e dois comparações }\end{array}$ & Sim & Não \\
\hline $\begin{array}{l}\text { Barros e cols. } \\
\qquad(2006)\end{array}$ & 2 & $\begin{array}{l}\text { Figuras abstratas, re- } \\
\text { forçadores comestíveis } \\
\text { e sons }\end{array}$ & Computador & $\begin{array}{l}\text { Discriminações simples e } \\
\text { matching por identidade com } \\
\text { atraso zero: um modelo e dois } \\
\text { comparações }\end{array}$ & Não & Sim \\
\hline $\begin{array}{l}\text { Wilkinson e } \\
\text { cols. (2009) }\end{array}$ & 5 & $\begin{array}{l}\text { Fotos, palavras ditadas } \\
\text { e pictogramas }\end{array}$ & Computador & $\begin{array}{l}\text { Matching simultâneo: um } \\
\text { modelo e três comparações }\end{array}$ & $\begin{array}{l}\text { Dois partici- } \\
\text { pantes }\end{array}$ & $\begin{array}{l}\text { Três partici- } \\
\text { pantes }\end{array}$ \\
\hline $\begin{array}{l}\text { O'Connor e } \\
\text { cols. (2009) }\end{array}$ & 15 & $\begin{array}{c}\text { Figuras, objetos e pala- } \\
\text { vras impressas }\end{array}$ & Papel & $\begin{array}{l}\text { Matching simultâneo: um } \\
\text { modelo e dois comparações }\end{array}$ & Não & Sim \\
\hline
\end{tabular}


No estudo clássico de Eikeseth e Smith (1992), conduzido com quatro autistas de alto funcionamento, foram ensinadas relações arbitrárias envolvendo apenas estímulos visuais (letras gregas maiúsculas relacionadas às suas correspondentes minúsculas $-\Xi-\xi / \Lambda-\lambda$; e as mesmas letras maiúsculas relacionadas a seus nomes impressos: $\Xi-\mathrm{Xi}$ / $\Lambda$ - Lambda) e em seguida foi avaliada a emergência de relações de equivalência. Na fase de ensino o número de tentativas de emparelhamento com o modelo variou entre 360 e 1546 até a aquisição do repertório de linha de base; na fase de testes (que requeria relacionar as letras minúsculas aos nomes impressos), nenhum dos participantes demonstrou consistentemente a formação de classes de estímulos equivalentes. Em fases seguintes do experimento, os pesquisadores ensinaram os participantes a nomear os estímulos visuais; nesta condição, eles demonstraram equivalência. Porém, na última fase do experimento, na qual foram ensinadas relações com novos estímulos, mas não foi requerida a nomeação dos estímulos, apenas dois dos quatro participantes demonstram formação de classes de estímulos equivalentes. Os pesquisadores concluíram que a nomeação pode ter favorecido a emergência de relações condicionais não diretamente ensinadas e atribuíram o elevado número de tentativas de treino a possíveis problemas no procedimento de ensino, que envolvia tarefas de matching típico, em que cada tentativa apresentava simultaneamente um estímulo modelo e dois estímulos de comparação.

Problemas no ensino de relações arbitrárias para autistas também foram encontrados no estudo de Dube e Mcllvane (1995), cujo objetivo era avaliar se estímulos reforçadores poderiam fazer parte de classes de equivalência. Nesse estudo, dois dos oito participantes tinham o diagnóstico de autismo. Os pesquisadores ensinaram aos participantes relações de identidade entre os estímulos abstratos A1, A2, B1 e B2 com uso de reforçadores específicos (reforçadores 1 e 2). Quando A1 era apresentado como modelo, a seleção de A1 era consequenciada com o reforçador 1 e quando A2 era o modelo, a seleção de $\mathrm{A} 2$ era consequenciada com um outro reforçador (reforçador 2). O ensino de identidade para o grupo B foi conduzido da mesma forma: ao apresentar B1 como modelo, a seleção de B1 era consequenciada com o reforçador 1 e quando B2 era o modelo, a seleção de B2 era consequenciada com o reforçador 2. Após aprenderem as relações de identidade, os participantes foram expostos a sondas das relações $\mathrm{AB}$ e $\mathrm{BA}$ (relações arbitrárias que não foram ensinadas diretamente). Quando A1 era apresentado como modelo, selecionar B1 era a resposta considerada correta, pelo fato de $\mathrm{A} 1$ e $\mathrm{B} 1$ terem sido relacionados ao mesmo reforçador 1. Quando A2 era modelo, a seleção de B2 era considerada correta por ambos os estímulos terem sido relacionados ao reforçador 2 . O mesmo era válido para as sondas das relações B1A1 e B2A2. Nessa situação, nenhum dos dois participantes com autismo demonstrou a emergência das relações $\mathrm{AB}$ e BA. Os autores, então, conduziram um segundo estudo com o objetivo de verificar se os participantes que não demonstraram emergência de relações com o uso de reforçadores específicos poderiam fazê-lo após o ensino direto de relações arbitrárias. Assim, foram ensinadas as relações arbitrárias $\mathrm{AB}$ (com os estímulos abstratos utilizados no primeiro estudo) e relações de identidade com um novo conjunto de estímulos $\mathrm{C}(\mathrm{C} 1$ e $\mathrm{C} 2)$, em procedimento semelhante ao do primeiro estudo. Para os participantes com autismo, os procedimentos de dica atrasada e fading foram utilizados no ensino das relações $\mathrm{AB}$, porém eles não aprenderam as relações. Em seguida foram conduzidas novas sessões de ensino de AB para um dos participantes com autismo, com procedimento de tentativa e erro e reforçamento diferencial, também sem sucesso.

Dados semelhantes com relação a problemas no ensino de relações condicionais com autistas foram apresentados por Vause e cols. (2005). O estudo teve por objetivo verificar se pessoas com repertório verbal mínimo poderiam formar classes de estímulos equivalentes e se o teste ABLA - Assessment of Basic Learning Abilities (Kerr, Meyerson, \& Flora, 1977) poderia predizer a formação ou não desse tipo de classe. Participaram do estudo cinco pessoas, uma delas com diagnóstico de autismo. Os participantes foram avaliados pelo teste ABLA e em outros testes padronizados de linguagem, como o Peabody Picture Vocabulary Test (Dunn \& Dunn, 1981) e a parte de comunicação da Vineland Adaptive Behavior Scale (Sparrow, Balla, \& Cicchetti, 1984). Além disso, foram conduzidas avaliações dos repertórios de tatos, mandos e imitação vocal dos participantes. Os estímulos utilizados eram palavras impressas, figuras e símbolos, apresentados em cartões de $10 \mathrm{~cm}$ x $15 \mathrm{~cm}$. Os participantes foram ensinados a relacionar palavras impressas a figuras (AB) e figuras a símbolos $(\mathrm{BC})$ em um procedimento de matching típico com uso de reforçamento diferencial para as respostas corretas e fading do tamanho dos estímulos de comparação incorretos. Inicialmente eram apresentados em tamanho normal o estímulo modelo e um dos estímulos de comparação (o estímulo designado como correto). O segundo estímulo de comparação era inicialmente menor e tinha seu tamanho aumentado gradativamente no decorrer das tentativas. Em seguida era introduzido o terceiro estímulo de comparação, também com aumento gradual de seu tamanho. Depois de estabelecida a linha de base de relações $\mathrm{AB}$ e BC, eram conduzidas as sondas de equivalência. Enquanto os participantes com desenvolvimento típico aprenderam a linha de base e formaram classes, o participante com autismo não aprendeu as relações $\mathrm{AB}$ e BC, mesmo após 1995 tentativas de treino, o que impossibilitou a realização de sondas de equivalência.

Carr e cols. (2000) investigaram a formação de classes de equivalência com pessoas que apresentavam repertório verbal mínimo e também utilizaram tarefas de matching típico simultâneo para o ensino de relações entre figuras abstratas. Dois adolescentes com autismo participaram do segundo experimento desse estudo. Os participantes passaram por avaliações padronizadas de linguagem e em seguida foram ensinados a relacionar os estímulos abstratos do conjunto A com os estímulos abstratos do conjunto $\mathrm{B}$, em tarefas de matching típico, com um estímulo modelo e dois estímulos de comparação apresentados simultaneamente. Depois de estabelecidas as relações $\mathrm{AB}$, um teste de simetria (relações BA) foi conduzido. Em seguida, foram ensinadas as relações $\mathrm{AC}$ e testadas as relações de simetria CA. Por fim, realizou-se uma sonda das relações emergentes $\mathrm{BC}$ e CB. Os dois participantes com autismo conseguiram aprender as relações 
de linha de base $\mathrm{AB}$ e $\mathrm{AC}$, porém o artigo não informa a quantidade de tentativas de treino até o critério de aprendizagem. Nas sondas de formação de classes um dos participantes demonstrou formação gradual de classes de equivalência e o outro não formou classes. Um detalhe interessante deste estudo é que o participante que apresentava o repertório verbal mais reduzido foi quem demonstrou emergência de classes de estímulos equivalentes.

Contrariamente aos insucessos (ou sucessos parciais) dos trabalhos descritos anteriormente, LeBlanc e cols. (2003) relataram completo sucesso com todos os participantes nas etapas de ensino de relações arbitrárias e nos testes das relações emergentes. O objetivo desse estudo, que utilizou metodologia baseada no paradigma de equivalência na pesquisa aplicada, foi ensinar habilidades de geografia e comparar três procedimentos de teste (dois que envolviam reforço e um conduzido sem reforço) para verificar relações emergentes. Os dois participantes com autismo precisaram de 100 a 180 tentativas de treino com tarefas de matching típico simultâneo, com um estímulo modelo e três de comparação, para aprenderem as relações arbitrárias envolvendo apenas estímulos visuais (mapas, nomes impressos de estados e nomes impressos de capitais). Ambos demonstrarem formação de classes de equivalência. Nesse estudo, o ensino de habilidades de caráter funcional e o emprego de estímulos que faziam parte do contexto social podem ter tido um papel relevante na aprendizagem das relações arbitrárias e na demonstração consistente de formação de classes de estímulos equivalentes.

Barros e cols. (2006), em um estudo que empregou um procedimento de ensino de discriminações simples e reversões sucessivas, também relataram sucesso no ensino e nos testes de relações emergentes. O objetivo desse trabalho foi verificar se consequências específicas para respostas na presença de conjuntos de estímulos (classes potenciais) poderiam fazer parte de classes de equivalência. Participaram do estudo duas crianças com autismo que apresentavam repertório verbal mínimo. Os participantes aprenderam discriminações simples entre pares de estímulos (A1A2; B1B2; $\mathrm{C} 1 \mathrm{C} 2$; D1D2): por exemplo, entre $\mathrm{A} 1$ (definido como $\mathrm{S}+$ ) e A2 (definido como S-). As seleções de A1, B1, C1 e D1 eram seguidas do reforçador 1 e do som 1. Depois de atingido o critério de aprendizagem, as funções eram revertidas: A1 passava a funcionar como S- e A2 como S+. Seleções de A2, B2, C2 e D2 eram seguidas sempre do reforçador 2 e do som 2. Após seguidas reversões das contingências, os estímulos do conjunto A eram apresentados em tarefas de matching por identidade com atraso zero: A1 era apresentado como estímulo modelo e após uma resposta de observação (tocar o estímulo na tela) ele era removido, dois estímulos de comparação eram imediatamente apresentados e a escolha do comparação idêntico ao modelo era reforçada. As contingências de reforço específico continuavam em vigor. O mesmo procedimento era realizado para o ensino dos conjuntos $\mathrm{B}$ e C. Foram realizadas sondas de relações emergentes AB, BA, $\mathrm{AC}$ e CA. Em seguida, testes de expansão de classe AD e DA foram conduzidos após os participantes serem submetidos ao ensino de discriminações simples com o novo conjunto de estímulos D. Os participantes aprenderam as discriminações simples e as relações condicionais de identidade sem grandes dificuldades. Os resultados dos testes de equivalência indicaram a formação de classes com os dois participantes do estudo. O único participante que foi submetido ao teste de expansão de classes (AD e DA) também demonstrou desempenhos consistentes, mesmo sem qualquer experiência prévia com matching por identidade com os estímulos do conjunto D.

Outro trabalho que envolveu relações de equivalência e participantes autistas foi descrito por Maguire e cols. (1994). Nesse estudo primeiramente foram estabelecidas relações condicionais arbitrárias entre alguns estímulos (figuras em preto e branco), por meio de tarefas de matching simultâneo com um estímulo modelo composto por dois itens e dois estímulos de comparação unitários; então foi verificada a emergência de relações simétricas entre eles. Em seguida, os pesquisadores examinaram métodos para ampliar o número de estímulos envolvidos nas classes, introduzindo um novo estímulo ao conjunto de itens do estímulo modelo composto, que passou a ter três itens. Participaram da pesquisa dois autistas adultos de 21 e 22 anos, além de quatro crianças com desenvolvimento típico. Os participantes com autismo aprenderam as relações arbitrárias e demonstraram a emergência de relações simétricas (não ensinadas diretamente). O sucesso nas relações aprendidas e testadas é evidência do potencial de aprendizagem dos participantes, mas os resultados quanto à formação de classes devem ser tomados com cautela, uma vez que foi medida apenas a simetria.

Dois trabalhos mais recentes sobre equivalência de estímulos com participantes com autismo têm em comum o fato de que os participantes apresentavam experiência prévia com matching típico. No primeiro estudo Wilkinson e cols. (2009) ensinaram relações auditivo-visuais envolvendo palavras ditadas e fotografias $(\mathrm{AB})$ e relações visuais-visuais envolvendo fotografias e pictogramas $(\mathrm{BC})$ e testaram a emergência das relações entre pictogramas e fotografias $(C B)$ e entre palavras ditadas e pictogramas (AC). As relações foram ensinadas pelo procedimento de exclusão (Dixon, 1977), em tarefas de matching típico, com um estímulo modelo e três estímulos de comparação. Participaram do estudo dez crianças com déficits cognitivos e de linguagem, cinco das quais tinham diagnóstico de autismo. Dessas cinco, três aprenderam as relações ensinadas e apresentaram emergência das relações testadas. Os outros dois participantes não atingiram o critério de aprendizagem das relações visuais-visuais e o artigo não relata o número de tentativas de treino realizadas.

No segundo estudo, O'Connor e cols. (2009) investigaram as relações entre desempenho nos testes de equivalência com a competência verbal dos participantes, o uso de antecedentes verbais durante as tarefas de matching típico, a nomeabilidade e a familiaridade dos estímulos empregados. Participaram do estudo quinze crianças com autismo com idades entre cinco e oito anos, além de três crianças com desenvolvimento típico. Os participantes foram submetidos a um procedimento que envolvia basicamente quatro fases: (1) treino/pré-teste de nomeação dos estímulos, (2) ensino de relações condicionais entre palavras impressas e objetos (AB), (3) ensino de relações condicionais entre objetos e figuras (BC) e (4) teste de relações emergentes entre palavras impressas e figuras, e vice-versa (AC e CA). 
Foram empregadas tarefas de matching típico, que apresentavam um estímulo modelo e dois estímulos de comparação. As quatro fases eram conduzidas em quatro estágios, apresentadas sequencialmente. Os quatro estágios diferiam quanto aos conjuntos de estímulos empregados: o Estágio 1 empregou estímulos nomeáveis, familiares e foi utilizado o antecedente verbal " este vai com o...". Somente neste estágio foi realizado o treino de nomeação para os estímulos do grupo $\mathrm{B}$ (objetos). O Estágio 2 empregou estímulos não nomeáveis e familiares, também com o uso de antecedentes verbais. O Estágio 3 fez uso de estímulos não nomeáveis e não familiares, com uso de antecedentes verbais. Por fim, o Estágio 4 apresentou estímulos não nomeáveis, não familiares e sem o uso de antecedentes verbais. Os participantes com autismo foram submetidos a dois experimentos: o primeiro deles contou com a participação de oito crianças e seguiu a ordem dos Estágios 1, 2, 3 e 4; o segundo contou com outras sete crianças com autismo e seguiu a ordem inversa de apresentação dos estágios (Estágios 4, 3, 2 e 1). Todas as crianças apresentaram formação de classes de equivalência, porém aquelas com uma menor competência verbal necessitaram de um número maior de tentativas de treino para estabelecer a linha de base e foi necessário um número maior de reapresentações de blocos de teste para que evidências de formação de classes fossem obtidas. Os pesquisadores também relataram que a formação de classes se deu mais prontamente com o uso de estímulos nomeáveis e familiares, em comparação com o uso de estímulos não nomeáveis e não familiares. Não foram relatados problemas no ensino de discriminações condicionais arbitrárias.

Apesar de escassos, estudos fundamentados no paradigma de equivalência envolvendo participantes autistas vêm sendo realizados no Brasil. Esse interesse crescente é evidenciado pelo número de teses e dissertações produzidas nos últimos anos, porém ainda não veiculadas em periódicos: Gomes, 2007; da Hora, 2009; Varella, 2009; Bagaiolo, 2009.

No estudo de Gomes (2007) o objetivo era ensinar leitura funcional a quatro crianças com autismo. Tarefas de matching adaptado $^{3}$ (Gomes \& de Souza, 2008), com três estímulos modelo e três estímulos de comparação apresentados simultaneamente, foram empregadas para ensinar relações condicionais arbitrárias entre figuras e palavras impressas. Os estímulos modelos eram compostos (figuras e palavras impressas) e os estímulos de comparação eram unitários (palavras impressas). O procedimento foi implementado com o uso de fichas em que os estímulos modelos compostos eram presos com velcro e o participante manipulava os estímulos de comparação, colocando cada um dos comparações sobre seus respectivos modelos; a tentativa era encerrada quando os três comparações eram deslocados para junto dos modelos. Os participantes também foram ensinados a nomear as figuras. Testes avaliaram a emergência de relações entre figuras

3 Este texto distingue entre o procedimento de matching típico ou padrão e o matching adaptado. No primeiro, cada tentativa apresenta um estímulo modelo e apenas um entre dois ou mais estímulos de comparação é considerado correto. No emparelhamento adaptado, cada tentativa apresenta simultaneamente dois ou mais estímulos modelos e o mesmo número de estímulos de comparação; a tarefa do participante é relacionar todos os estímulos, cada comparação a um modelo específico (Gomes $\&$ de Souza, 2008). e palavras impressas, a nomeação de palavras impressas e a identificação de palavras impressas (ou reconhecimento de palavras, que consistia em escolher, entre três comparações, uma palavra impressa correspondente à palavra ditada). Os participantes adquiriam as relações de linha de base, apresentaram relações emergentes que documentaram a formação de classes de estímulos equivalentes e apresentaram comportamento textual emergente.

No estudo de da Hora (2009) o objetivo foi avaliar se relações emergentes poderiam ser observadas em participantes autistas utilizando-se um procedimento go/no-go, que difere do matching típico, com estímulos compostos constituídos por figuras abstratas. Foram empregados oito pares de estímulos compostos, sendo quatro positivos ( $\mathrm{S}+$, isto é, a resposta na sua presença era seguida de consequência) e outros quatro negativos (S-, ou extinção). Cada tentativa apresentava um estímulo composto e a tarefa consistia em responder em tentativas com $\mathrm{S}+$ e não responder em tentativas com S-. No Estudo 1 apenas um de quatro participantes atingiu critério de aprendizagem no treino das relações condicionais e apresentou desempenhos emergentes. No Estudo 2, após modificação no procedimento de ensino, de modo que a duração dos estímulos compostos negativos (S-) era inicialmente curta e foi gradualmente aumentada, apenas uma de três crianças com autismo atingiu o critério de aprendizagem para a realização dos testes de simetria, mas não exibiu desempenhos emergentes.

Varella (2009) investigou a formação de classes de equivalência em pessoas autistas com repertório verbal reduzido. Quatro participantes que haviam demonstrado identidade generalizada foram submetidos ao ensino de discriminações condicionais com estímulos visuais abstratos. Foram ensinadas as relações $\mathrm{AB}$ e $\mathrm{CB}$ (para os participantes $\mathrm{P}$, $\mathrm{P} 4$ e P5) e as relações $\mathrm{AB}, \mathrm{CB}$ e $\mathrm{DB}$ (para o participante $\mathrm{P} 2$ ). Os participantes $\mathrm{P} 2$ e $\mathrm{P} 3$ adquiriram as discriminações condicionais arbitrárias e demonstraram formação de classes equivalentes. P4 e P5 não adquiriram as discriminações condicionais, mesmo com o emprego de diversos procedimentos remediativos.

Bagaiolo (2009, Estudo 2) documentou a formação de classes de equivalência e a emergência do controle por unidades verbais mínimas com uma adolescente com autismo a quem foram ensinadas relações condicionais arbitrárias auditivo-visuais entre palavras ditadas, figuras e palavras impressas. A estrutura de treino utilizada foi $\mathrm{AB}$ e $\mathrm{AC}$, com sondas das relações emergentes $\mathrm{BC}$ e $\mathrm{CB}$. A participante adquiriu as discriminações condicionais e demonstrou emergência das relações de equivalência. Apesar disso, foi relatada uma grande dificuldade em se estabelecer os repertórios de linha de base e foi necessária uma série de procedimentos remediativos para promover a aquisição das discriminações. Após as sondas de relações emergentes, a participante foi submetida a um treino de construção de anagramas envolvendo as palavras empregadas no treino. Após o ensino da tarefa de compor as palavras por meio da seleção de sílabas, foram conduzidas sondas que consistiam em tarefas de matching envolvendo novas palavras formadas pela recombinação das sílabas presentes nas palavras do treino. A participante também demonstrou emergência de controle por unidades verbais mínimas. 


\section{Variáveis relevantes e perspectivas de investigação futura}

Os dados dos estudos que investigaram a formação de classes de estímulos equivalentes com participantes com autismo indicam que as dificuldades não incidem somente na formação de classes, que poderia estar relacionada às alterações na linguagem inerentes ao autismo; antes, como apontado em muitos dos trabalhos, ocorrem dificuldades primárias na aprendizagem de relações condicionais arbitrárias, o que também tem sido encontrado em estudos com crianças muito jovens ou com retardo mental (Pilgrim \& cols., 2000). Assim, sem que o indivíduo aprenda pelo menos duas relações condicionais arbitrárias diretamente ensinadas, a depender do procedimento de ensino adotado, torna-se inviável a condução dos testes críticos de emergência de relações não diretamente ensinadas que demonstrem a formação de classes de estímulos equivalentes (Sidman \&Tailby, 1982; Sidman, 1994).

O recurso utilizado para o ensino de relações arbitrárias na maioria dos estudos descritos foi o emparelhamento com o modelo típico ou matching típico, em que cada tentativa apresenta um estímulo modelo e dois ou mais estímulos de comparação, um dos quais é definido como correto, isto é, sua escolha é reforçada (de Rose, 1998).

Dificuldades no ensino de relações condicionais arbitrárias com participantes autistas por meio de tarefas de matching típico têm sido encontradas também em investigações sobre controle de estímulos que não envolveram equivalência. Em um estudo conduzido por Williams, Perez-González e Queiroz (2005) foram necessárias 792 tentativas de treino para ensinar a um adolescente com autismo relações auditivo-visuais entre as cores branca e preta e seus respectivos nomes ditados. No estudo de Kelly, Green e Sidman (1998), que visava ensinar e avaliar relações de identidade entre estímulos visuais e relações arbitrárias auditivo-visuais com uma criança com autismo, verificou-se que o desempenho do participante nas tarefas de matching variava de acordo com a maneira pela qual as tarefas eram apresentadas (por computador ou em papel) e também, de acordo com o número de estímulos de comparação presentes. Os pesquisadores concluíram que o baixo desempenho da criança em algumas tarefas provavelmente resultou dos procedimentos de ensino e teste utilizados.

Uma alternativa ao matching típico para o ensino de relações entre estímulos com autistas é utilizada pelo programa TEACCH (Treatment and Education of Autistic and Communication Handicapped Children; Mesibov, Schopler, \& Hearsey, 1994). A adaptação proposta por esse programa leva em consideração aspectos da aprendizagem específicos do autismo e, diferentemente do matching típico, emprega um matching adaptado, que apresenta o mesmo número de estímulos modelos e comparações em cada tentativa. Dessa forma, se a tentativa apresenta três estímulos de comparação, há também três estímulos modelo e a tarefa do participante é relacionar cada comparação ao seu respectivo modelo. A principal justificativa para esta adaptação é que pessoas com autismo parecem responder melhor a tarefas que sejam visualmente lógicas, ou seja, tarefas em que a própria organização dos estímulos indique ao aprendiz o que ele deve fazer, sem a necessidade de muitas instruções verbais. O fato de emparelhar os estímulos um a um, até completar todos os pares, torna a atividade mais óbvia, indicando visualmente o término da tarefa, uma vez que nenhum estímulo fica "sobrando" (ou sem um par), diferentemente do matching típico em que a tentativa é encerrada após a escolha de apenas um dos estímulos de comparação.

Esses dois tipos de emparelhamento foram investigados por Gomes e de Souza (2008), que avaliaram o desempenho de vinte pessoas com autismo em tarefas de emparelhamento com o modelo por identidade, sob a organização característica tanto do matching adaptado quanto do matching típico. $\mathrm{O}$ objetivo era apenas avaliar o desempenho dos participantes; portanto, as relações de identidade não foram ensinadas. Os resultados indicaram que a média de acertos foi significativamente maior nas tarefas de matching adaptado, principalmente para participantes com escores mais baixos. Os dados revelaram acentuada variabilidade inter-individual; para os participantes que dominavam a tarefa, o tipo de arranjo dos estímulos mostrou-se irrelevante, porém os que apresentavam dificuldades alcançaram uma porcentagem de acertos muito maior sob o matching adaptado. Esses resultados foram confirmados e estendidos pelo estudo de Gomes (2007, Estudo 2), no ensino de relações arbitrárias. Portanto, evidências experimentais sugerem que o matching adaptado pode ser uma alternativa para o ensino de relações arbitrárias entre estímulos visuais para participantes com autismo, estabelecendo a linha de base necessária para a avaliação subsequente da formação de classes de estímulos equivalentes.

Além da limitação das tarefas de matching típico para o ensino de relações arbitrárias com participantes autistas, os estudos descritos apontaram outras variáveis importantes que devem ser consideradas na busca por procedimentos eficazes. No estudo de Eikeseth e Smith (1992) os participantes formaram classes de equivalência somente após o ensino de nomeação dos estímulos, o que sugere que dizer o nome do estímulo pode ser um facilitador da emergência de relações não ensinadas. A questão da necessidade da nomeação enquanto resposta verbal que exerce uma função mediadora (Horne \& Lowe, 1996) para a formação de classes de equivalência é controvertida, mas parece haver um consenso de que ela facilite a emergência de relações não ensinadas diretamente (Randell \& Remington, 1999; Sidman, 1994). Do um ponto de vista educacional, os dados disponíveis indicam que ensinar a nomear os estímulos, concomitante ou alternadamente com tarefas de discriminação condicional, pode ser uma estratégia interessante para aumentar a probabilidade de que aprendizes com autismo demonstrem formação de classes de estímulos equivalentes e desenvolvam comportamentos de caráter simbólico.

Outra variável que pode estar relacionada aos resultados negativos em testes de equivalência é a estrutura dos treinos (Fields, Adams, Verhave, \& Newman, 1990). Saunders e Green (1999) argumentaram que a variabilidade encontrada nos resultados de testes de equivalência pode estar relacionada ao arranjo do ensino das relações arbitrárias. Os autores distinguem três tipos possíveis de estruturas: (1) quando os estímulos modelo são relacionados a dois ou mais conjuntos de estímulos de comparação (modelo como nódulo), por exemplo, ensinar $\mathrm{AB}$ e depois $\mathrm{AC}$; (2) quando os estímulos 
de comparação são relacionados a dois ou mais conjuntos de modelos (comparação como nódulo), por exemplo, ensinar $\mathrm{AB}$ e, em seguida, $\mathrm{CB}$; e por fim (3) a estrutura em série linear, em que são ensinadas as relações $\mathrm{AB}$ e BC. Estas diferentes organizações resultam em diferentes apresentações dos estímulos modelo e de comparação durante as etapas de ensino, que podem ser fundamentais para os aprendizes estabelecerem as discriminações simples envolvidas no procedimento. Entre os artigos revisados, os de Carr e cols. (2000) e Eikeseth e Smith (1992) utilizaram a estrutura "modelo como nódulo" (AB e AC); os estudos de LeBlanc e cols. (2003), O'Connor e cols. (2009), Vause e cols. (2005) e Wilkinson e cols. (2009) utilizaram a estrutura em série linear (AB e BC); o estudo de Maguire e cols. (1994) foi o único a apresentar uma estrutura com comparação como um nódulo. Estudos que investigaram o papel da estrutura de treino com outros tipos de participantes, principalmente universitários (Fields e cols., 1990), sugerem que a estrutura de treino pode ser uma variável importante a ser considerada e a depender dos objetivos do pesquisador/educador, uma estrutura pode ser mais apropriada que outra. Segundo Saunders e Green (1999), a emergência de relações de equivalência é mais provável em estruturas de comparação como nódulo, mesma estrutura utilizada no estudo de Varella (2009), em que se observou a emergência de relações de equivalência em dois dos quatro participantes com autismo (os outros dois participantes não adquiriram a linha de base).

Os estímulos utilizados nas relações ensinadas também são variáveis relevantes que merecem atenção. No estudo de LeBlanc e cols. (2003) participantes com autismo não mostraram dificuldades em aprender relações arbitrárias entre mapas, nomes impressos de estados e nomes impressos de capitais. De modo semelhante, no estudo de O'Connor e cols. (2009) a formação de classes se deu mais prontamente com o uso de estímulos nomeáveis e familiares, em comparação com o uso de estímulos não nomeáveis e não familiares. Provavelmente o uso de estímulos familiares e menos "abstratos" pode ter aumentado o engajamento e o acerto nas tarefas.

Outro caminho interessante, apontado por Dube e McIlvane (1995) e ressaltado por Barros e cols. (2006), é a possibilidade de formação de classes de estímulos equivalentes a partir do estabelecimento de relações mais simples, como o ensino de discriminações simples e matching por identidade com apresentações de consequências específicas para as classes. Debert, Matos e McIlvane (2007) e da Hora (2009) também apresentam novas possibilidades que merecem mais investigações com o procedimento go/no-go para estabelecer classes de estímulos equivalentes.

$\mathrm{O}$ conjunto de dados disponíveis a respeito da formação de classes de estímulos equivalentes por participantes autistas indica a necessidade de novas investigações acerca de procedimentos de ensino que favoreçam a aprendizagem de relações arbitrárias e possibilitem avaliar a formação de classes de estímulos equivalentes. Estudos sobre equivalência com participantes autistas podem ser importantes para aumentar a compreensão sobre sua aprendizagem e linguagem. Os resultados de pesquisas nessa área poderiam ainda, ampliar os modelos existentes de intervenção com autistas, principalmente no que se refere aos conteúdos que envolvem linguagem, possibilitando assim que pessoas com esse diagnóstico compreendam o meio social e interajam com outras pessoas de maneira mais ativa e funcional.

\section{Referências}

Associação Americana de Psiquiatria. (2002). Manual diagnóstico e estatístico de transtornos mentais - Texto revisado. $4^{\text {a }}$ edição. Porto Alegre: Artes Médicas.

Bagaiolo, L. (2009). Padrões de aquisição de discriminação condicional durante a emergência do controle por unidades verbais mínimas em leitura em crianças com autismo e desenvolvimento típico. Tese de Doutorado, Universidade de São Paulo, São Paulo.

Barros, R. S., Lionello-DeNolf, K. M., Dube, W. V., \& McIlvane, W. J. (2006). A formação de classes de equivalência via pareamento por identidade e discriminação simples com consequências específicas para as classes. Revista Brasileira de Análise do Comportamento, 2(1), 79-92.

Carr, D., Wilkinson, K. M., Blackman, D., \& McIlvane, W. J. (2000). Equivalence classes in individuals with minimal verbal repertoires. Journal of the Experimental Analysis of Behavior, 74(1), 101-114.

Catania, A. C. (1999). Aprendizagem: Comportamento, linguagem e cognição. Porto Alegre: Artmed.

da Hora, C. L. (2009). Procedimento go/no-go com estímulos compostos e relações condicionais emergentes em crianças com autismo. Dissertação de Mestrado, Universidade de São Paulo, São Paulo.

de Rose, J. C. (1998). Equivalência de estímulos: problemas atuais de pesquisa. Anais da XVIII Reunião Anual de Psicologia (pp.19-32). Ribeirão Preto: Sociedade de psicologia de Ribeirão Preto.

de Rose, J. C. (2004). Emparelhamento com modelo e suas aplicações. Em C. N. de Abreu, \& H. J. Guilhardi (Orgs.). Terapia comportamental e cognitivo-comportamental: Práticas clínicas (pp. 215-225). São Paulo: Roca.

de Rose, J. C., de Souza, D. G., \& Hanna, E. S. (1996). Teaching reading and spelling: Exclusion and stimulus equivalence. Journal of Applied Behavior Analysis, 27, 451-469.

Debert, P., Matos, M. A., \& McIlvane, W. J. (2007). Conditional relations with compound abstract stimuli using a go/no-go procedure. Journal of the Experimental Analysis of Behavior, 87, 89-96.

Dixon, L. S. (1977). The nature of control by spoken words over visual stimulus selection. Journal of the Experimental Analysis of Behavior, 27, 433-442.

Dube, W. V. (1996). Teaching discriminations skills to persons with mental retardation. Em C. Goyos, M. A. Almeida, \& D. G. de Souza (Orgs.), Temas em Educação Especial (pp. 73-96). São Carlos: EDUFSCar.

Dube, W., \& McIlvane, W. (1995). Stimulus-reinforcer relations and emergent matching to sample. The Psychological Record, 45, 591-612.

Dunn, L.M., \& Dunn, L.M. (1981). Peabody Picture Vocabulary Test: Revised. Circle Pines: American Guidance Service.

Eikeseth, S., \& Smith, T. (1992). The development of functional and equivalence classes in high-functioning autistic children: The role of naming. Journal of the Experimental Analysis of Behavior, 58, 123-133. 
Fields, L., Adams, B. J., Verhave, T., \& Newman, S. (1990). The effects of modality on the formation of equivalence classes. Journal of the Experimental Analysis of Behavior, 53(3), 345-358.

Filipek, P. A., Accardo, P. J., Baranek, G. T., Cook Jr., E. H., Dawson, G., Gordon, B., \& cols. (1999). The screening and diagnosis of autistic spectrum disorders. Journal of Autism and Developmental Disorders, 29, 439-484.

Green, G., Sigurdardottir, Z. G., \& Saunders, R. R. (1991). The role of instructions in the transfer of ordinal functions through equivalence classes. Journal of the Experimental Analysis of Behavior, 55, 287-304

Gomes, C. G. (2007). Desempenhos emergentes e leitura funcional em crianças com transtornos do espectro autístico. Dissertação de Mestrado, Universidade Federal de São Carlos, São Paulo.

Gomes, C. G., \& de Souza, D. G. (2008). Desempenho de pessoas com autismo em tarefas de emparelhamento com o modelo por identidade: efeitos da organização dos estímulos. Psicologia: Reflexão e Crítica, 21(3), 412-423.

Horne, P. J., \& Lowe, F. C. (1996). On the origins of naming and other symbolic behavior. Journal of the Experimental Analysis of Behavior, 65(1), 185-241.

Kanner, L. (1943). Affective disturbances of affective contact. Nervous Child, 2, 217-250.

Kelly, S., Green, G., \& Sidman, M. (1998). Visual identity matching and auditory-visual matching: A procedural note. Journal of Applied Behavior Analysis, 31(2), 237-243.

Kerr, N., Meyerson, L., \& Flora, J. (1977). The measurement of motor, visual and auditory discrimination skills. Rehabilitation Psychology, 24, 95-112.

LeBlanc, L., Miguel, C. F., Cumming, A., Goldsmith, T., \& Carr, J. E. (2003). The effects of three stimulus-equivalence testing conditions on emergent U. S. geography relations of children diagnosed with autism. Behavioral Interventions, 18, 279-289.

Lovaas, O. I. (2003). Teaching individuals with developmental delays. Austin, Tx.: PRO-ED.

Maguire, R. W., Stromer, R., Mackay, H. A., \& Demis, C. A. (1994). Matching to complex samples and stimulus class formation in adults with autism and young children. Journal of Autism and Developmental Disorders, 24, 753-772.

Mesibov, G. B., Schopler, E., \& Hearsey, A. (1994). Structured teaching. Em E. Schopler, \& G. B. Mesibov (Eds.), Behavior issues in autism (pp. 195-210). New York: Plenum Press.

O’Connor, J., Rafferty, A., Barnes-Holmes, D., \& Barnes-Holmes, Y. (2009). The role of verbal behavior, stimulus nameability and familiarity on the equivalence performances of autistic and normally developing children. Psychological Record, 59(10), 53-74.

Pilgrim, C., Jackson, J., \& Galizio, M. (2000). Acquisition of arbitrary conditional discriminations by young, normally developing children. Journal of the Experimental Analysis of Behavior, 73, 177-194.
Prado, P. S. T., \& de Rose, J. C. (1999). Conceito de número: uma contribuição da análise comportamental da cognição. Psicologia: Teoria e Pesquisa, 15(3), 227-235.

Randell, T., \& Remington, B. (1999). Equivalence relations between visual stimuli: The functional role of naming. Journal of the Experimental Analysis of Behavior, 71(3), 395-415.

Saunders, R., \& Green, G. (1999). Discrimination analysis of training-structure effects on stimulus equivalence outcomes. Journal of the Experimental Analysis of Behavior, 72, 117-137.

Sidman, M. (1971). Reading and auditory-visual equivalences. Journal of Speech and Hearing Research, 14, 5-13.

Sidman, M. (1994). Equivalence relations and behavior: A research story. Boston: Authors Cooperative.

Sidman, M., \& Cresson, O. (1973). Reading and crossmodal transfer of stimulus equivalencies in severe mental retardation. American Journal of Mental Deficiency, 77, 515-523.

Sidman, M., \& Tailby, W. (1982). Conditional discrimination vs. matching to sample: An expansion of the testing paradigm. Journal of the Experimental Analysis of Behavior, 37, 5-22.

Sparrow, S., Balla, D. A., \& Cicchetti, D. V. (1984). The Vineland Adaptive Behavior Scales. Circle River, MN: American Guidance Service.

Sundberg, M. L., \& Partington, J. W. (1998). Teaching language to children with autism or other developmental disabilities. Danville, CA: Behavior Analysts, Inc.

Varella, A. A. B. (2009). Ensino de discriminações condicionais e avaliação de desempenhos emergentes em autistas com reduzido repertório verbal. Dissertação de Mestrado, Universidade Federal de São Carlos, São Paulo.

Vause, T., Martin, G. L., Yu, C. T., Marion, C., \& Sakko, G. (2005). Teaching equivalence relations to individuals with minimal verbal repertoires: Are visual and auditory-visual discriminations predictive of stimulus equivalence? The Psychological Record, 55(2), 197-218.

Wilkinson, K. M., Rosenquist, C., \& McIlvane, W. J. (2009). Exclusion learning and emergent symbolic category formation in individuals with severe language impairments and intellectual disabilities. The Psychological Record, 59, 187-206.

Williams, G., Pérez-González, L. A., \& Queiroz, A. (2005). Using a combined blocking procedure to teach color discrimination to a child with autism. Journal of Applied Behavior Analysis, $38,555-558$. 